\section{SCIDoC \\ Publishers}

(n)

ISSN 2332-2748

\title{
Use of a Topical Spray Made of Essential Oils and Essential Fatty Acids from Plant Extracts for the Treatment of Microbial Infections in Dogs: An Opened Multicentric Study of 47 Cases
}

Research Article

Bensignor $\mathrm{E}^{1^{*}}$, Vidémont $\mathrm{E}^{2}$

${ }^{1}$ Dermatology Referral Service, Rennes-Cesson, Paris, Nantes, France.

${ }^{2}$ CHV Saint Martin, Saint Martin Bellevue, France.

Abstract

Bacterial pyoderma and Malassezia dermatitis are frequent dermatosis in dogs, for which the use of systemic courses of respectively antibiotics and/or anti fungals is needed. However, as the risk of emergence of antimicrobial resistance in dermatology is growing, the use of topical antiseptics or antimicrobials is highly recommended. This study describes the value of a topical spray made of essential fatty acids and essential oils from plant origin to help treating antimicrobial infections in dogs. 47 dogs suffering from skin infection diagnosed on the basis of clinical and cytological signs were included and treated with a twice daily application of the spray. Follow-up visits were performed after 10 and 21 days. The rate of satisfaction was $90 \%$ for veterinarians and $89 \%$ for owners. Extent of lesions decreased by $23 \%$ and $37 \%$ after respectively 10 and 21 days. Severity of lesions decreased by $42 \%$ and $61 \%$ after respectively 10 and 21 days. Few side effects were reported. These results suggest that the use of a topical antimicrobial agent can help in treating bacterial or yeast infection in dogs.

Keywords: Bacterial Pyoderma; Antimicrobials; Essential Fatty Acids; Spot-On.

\section{Introduction}

Microbial infections (bacterial pyoderma and Malassezia dermatitis) are one of the most frequent diseases in canine dermatology [1]. Their treatment usually necessitates the use of systemic antimicrobial drugs such as respectively antibiotics and antifungals [2]. However, antimicrobial resistance has become a recent issue in veterinary dermatology and it is therefore currently recommended, when possible, to avoid the use of such products [2-4]. The topical application of antiseptic products, initially considered only as a valuable adjunctive therapy, has been recently demonstrated in preliminary studies to be of benefit to either prevent the use or reduce the total dose of antibiotics for bacterial pyoderma [5-9]. For Malassezia dermatitis, it has also been demonstrated for localized cases that topical application of antifungal agents was as effective as the oral administration of ketoconazole both for reducing clinical signs and yeasts counts [10]. However, even if potentially effective as demonstrated in clinical trials in referral centres, it is not known if this approach is considered as efficacious and satisfactory by owners and veterinarians in general practice. The aim of this opened non controlled study was to evaluate the value of a novel spray, containing essential oils and essential fatty acids from plant extracts with antimicrobial properties in the treatment of staphylococcal and/or Malassezia pachydermatis infections in dogs and to assess the evaluation of this products by owners and veterinarians.

\section{Material and Methods}

This was an opened multicentre study conducted in 22 veterinary clinics in various countries (France 1, Germany 5, Japan 1, Taiwan 1, South Africa 2, USA 1, China 8, Malaysia 1, Spain 2). Private owned dogs suffering from an infectious dermatosis (either bacterial pyoderma and/or Malassezia dermatitis) were treated with a spray containing essential plants in an uncontrolled trial. Written owner consent was obtained before including the animals when needed by regulatory rules depending on the countries.

\footnotetext{
*Corresponding Author:

E Bensignor,

Dermatology Referral Service, 35510 Rennes-Cesson, 75003 Paris, 44000 Nantes- France.

Tel: 33299833130

E-mail: emmanuel.bensignor@wanadoo.fr

Received: February 13, 2017

Accepted: March 21, 2017
}

Published: March 23, 2017

Citation: Bensignor E, Vidémont E (2017) Use of a Topical Spray Made of Essential Oils and Essential Fatty Acids from Plant Extracts for the Treatment of Microbial Infections in Dogs: An Opened Multicentric Study of 47 Cases. Int J Vet Health Sci Res. 5(2), 161-164. doi: http://dx.doi.org/10.19070/2332-2748-1700033

Copyright: Bensignor $\mathbf{E}^{\odot}$ 2017. This is an open-access article distributed under the terms of the Creative Commons Attribution License, which permits unrestricted use, distri bution and reproduction in any medium, provided the original author and source are credited. 


\section{Dogs}

Dogs of any age, breed and sex presented to a veterinarian with clinical signs compatible with either superficial bacterial pyoderma or Malassezia dermatitis were included in the study. Lesions consistent with bacterial pyoderma included papules, pustules, epidermal collarettes and/or crusts/scales. Lesions consistent with Malassezia dermatitis included erythema, greasy seborrhea and/or scaling. Cytological examination consistent with bacterial pyoderma included the presence of cocci phagocytosed by neutrophils on microscopical examination and cytological examination consistent with Malassezia dermatitis included the presence of numerous (at least 5) yeasts per field at high power (Gx100). Dogs suffering from deep pyodermas (presence of furoncles, erosions and/or ulcers) were excluded as they were unlikely to benefit from the use of the topical product alone. Dogs suffering from a parasitic skin disease (eg demodicosis, sarcoptic mange, flea infestation) were excluded by skin scrapings, flea combing and when needed a serological examination.

To be included, dogs should not have been treated in the past 7 days with any topical product and systemic antibiotics. The owners agreed to perform the treatment and to comply with the visits and signed an informed consent if needed by regulatory authorities.

\section{Clinical Scoring}

At inclusion, the Investigator collected a patient history and completed a standard physical and dermatological examination. The diagnosis of bacterial pyoderma and/or Malassezia dermatitis was performed and the lesions were scored depending on their extension and their severity. The extent was qualified as 0 (no lesion), 1 (one lesion), 2 (2 to 3 lesions) and 3 (more than 3 lesions). The severity was scored as 0 (absence), 1 (mild), 2 (moderate), 3 (rather severe) and 4 (severe). Veterinary and owner satisfactions were assessed as 0 (unsatisfied), 1 (moderately satisfied), 2 (satisfied) and 3 (very satisfied).

\section{Treatment}

Each dog was treated with the application of the tested product Dermoscent $^{\circledR} \quad$ PYOclean $^{\circledR}$ Spray, Laboratoire de DermoCosmetique Animale, Castres, France) directly on the lesions twice daily. The continuation of use of an insecticidal therapy (product and dosage to be decided by the veterinarian) was needed to prevent a flea infestation during the trial period. Follow-up visits were scheduled after 10 and 21 days. At each visit a clinical and cytological evaluation was performed and the owners were asked about any side effect and about their satisfaction.

\section{Data Analysis}

Data were expressed as mean, standard deviation (SD) and range. For each clinical parameter and lesion score, the Wilcoxon signedrank test was used to compare the scores of both groups at each visit.

Clinical efficacy was calculated as the percentage of change between each post-treatment and pre-treatment total scoring count using the formula: \% Reduction $=[1-($ Post $T x$ value $\div$
Pre'Tx value)] x 100. Statistical significance was declared when P $<0.05$. The primary software was GraphPad Prism version 5.00 for Windows (GraphPad Software, San Diego California USA).

\section{Results}

\section{Study Population}

22 veterinary clinics included $47 \mathrm{dogs}$ from South Africa (2 clinics, 2 dogs), USA ( 1 clinic, 1 dog), Germany ( 5 clinics, 20 dogs), Japan (1 clinic, 3 dogs), France (1 clinic, 2 dogs), China (8 clinics, 8 dogs), Malaysia (1 clinic, 6 dogs), Taiwan (1 clinic, 3 dogs) and Spain (2 clinics, 2 dogs). 23 different breeds were represented, with French Bouledogues, Labrador retrievers Poodles and crossbreeds over represented ( 4 cases each). 23 females (12 intact and 11 neutered) and 24 males (16 intact and 8 neutered), aged from 8 months to 17 years (median 4.7 years), weighing from 1.8 to 53.6 kgs (median $17.5 \mathrm{kgs}$ ) were included.

\section{Clinical Scores}

All the dogs completed the study. At inclusion various lesions were present: erythema (19 cases), papules (14 cases) pustules ( 9 cases), epidermal collarettes (5 cases), scales/crusts (14 cases), greasy seborrhea (6 cases), alopecia (6 cases), hot spot (4 cases), oozing/erosions (9 cases), lichenification/hyperpigmentation (7 cases). Lesions were localized on the head and ears (19 cases), the feet (10 cases), the thorax/axillary area (13 cases), the abdominal/ inguinal area (21 cases), the neck (5 cases) and/or the back (11 cases). At inclusion, extent score of the lesions was 2.062 (SD 0.8418 ) and this parameter decreased throughout the study period (less $22.55 \%$ after 10 days, $\mathrm{p}<0.05 \%$, Wilcoxon signed rank test; less $37.15 \%$ after 21 days, $\mathrm{p}<0.001 \%$, Wilcoxon signed rank test) (Figure 1). Severity scores also significantly decreased with time with an improvement of $41.90 \%$ after 10 days and $61.01 \%$ after 21 days $(p<0.01$, Wilcoxon signed rank test) (Figure 2).

\section{Owner and Veterinarian Appreciation}

Owners were satisfied or very satisfied in $89 \%$ of the cases. Veterinarians were satisfied or very satisfied in $90 \%$ of the cases.

\section{Side Effects}

Only few side effects (burning sensation, pruritus, erythema) at the site of application were reported during the study, most of them being benign and spontaneously regressing after treatment withdrawal $(5 / 47 \mathrm{dogs})$.

\section{Discussion}

The aim of our study was to confirm that the use of a nonantibiotic spray containing essential oils and essential fatty acids from plant extracts, demonstrated previously to have antimicrobial properties [5], was of interest in the management of bacterial pyoderma and/or Malassezia dermatitis in dogs in the field. Bacterial and Malassezia overgrowth are very common in general practice [1-4]. They are usually associated with an underlying disease, such as allergy. Beside the control of the primary disease, their treatment depends on the extent and severity of clinical signs and the possibility to use topical treatments [4]. Topical 
Figure 1. Extent of Lesions before Treatment and after 10 and 21 days of Treatment.

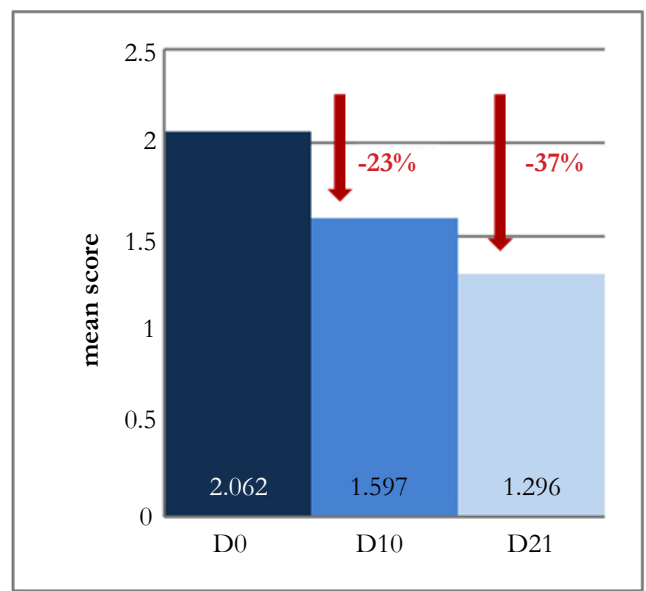

Figure 2. Severity of Lesions before Treatment and After 10 and 21 Days of Treatment.

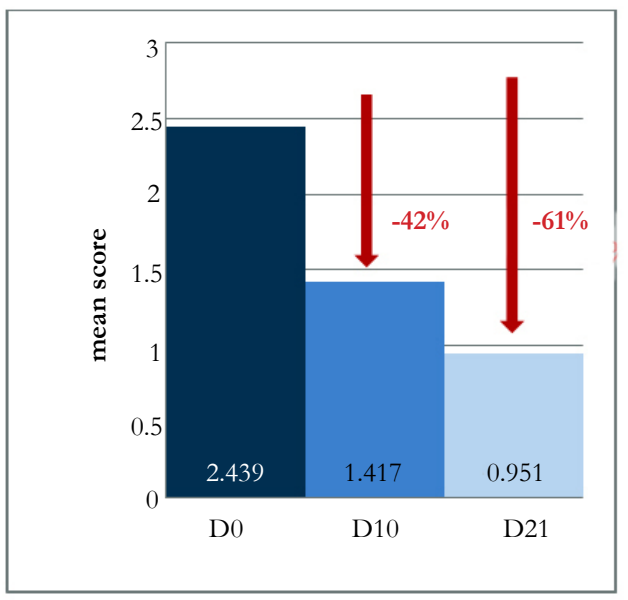

treatment is not only an interesting adjunctive therapy but can avoid, in some cases, the use of systemic treatments notably antibiotics [3]. Due to the emergence of methicillin-resistant Staphylococcus pseudintermedius (MRSP) and other multi-drug resistant staphylococci in veterinary dermatology, the value of alternative options to antibiotics has been the scope of various studies and reviews, both in vivo and in vitro, but mainly using biocide products such as chlorhexidine [7-9]. The tested product, Dermoscent ${ }^{\circledR}$ PYOclean ${ }^{\circledR}$ spray is composed of $\mathrm{N}$-acetylcysteine (NAC) associated with lavendin, manuka and hempseed oil. An oligosaccharidic prebiotic completes the formula. NAC is a molecule derived from the amino acid cysteine, commonly used as antioxidant and free-radical scavenging, because it increases cellular production of glutathione. It has also interesting properties in the dermatology field as the ability to prevent biofilm formation, treat microbial infections and restore skin integrity $[11,12]$. Biofilm is a multicellular microbial community of one or more microorganisms extremely capable to self-reproduce on biological surfaces. It is a complex ecosystem that provides extra protection for the sessile bacteria cells inside the biofilm from external factors such as dessication, the host immune response and antimicrobials. Systemic antibiotics are often ineffective because of their slow and incomplete penetration into the deepest layers of biofilm [4]. The control of biofilm growth is therefore a really challenging target as biofilm formation may be involved in many chronic and relapsing infections, including skin infections.
In vitro and in vivo studies have demonstrated that NAC is effective in inhibiting biofilm formation, disrupting preformed biofilms and reducing bacterial viability in biofilms [11]. Manuka oil, another component of the spray, can also interfere with biofilm formation [13], and has a synergistic action with NAC. NAC has shown antibacterial effects against a variety of Gram-negative and Gram-positive bacteria [12]. It has a synergistic activity with lavendin, manuka and hempseed oil, that have all demonstrated antibacterial activity, both in vitro and in vivo, notably against Gram-positive bacteria including some antibiotic-resistant strains [13-15]. These essential oils are also efficacious against yeasts [16, 17]. This is important because commonly used antiseptic products in dermatology have mainly activity against bacteria. The use of a product combining activity against bacteria and Malassezia is preferable. Finally, the spray may also be effective by improving skin integrity and skin barrier function. In effect, beside antiinflammatory effect and promotion of wound healing [18] NAC can decrease transepidermal water loss and thus contribute to restore the skin barrier [19]. Hempseed oil is a rich and balanced source of omega- 6 and omega-3 polyunsaturated fatty acids [20] and it is now well recognized that polyunsaturated fatty acids are of great importance for the integrity of skin barrier [21]. The restoration of skin barrier helps to improve the wellness of the animal by decreasing skin xerosis and itching and contribute to limit microbial proliferations as skin is less prone to secondary infections. These combined properties explain the good results 
obtained in this study, both on lesions directly linked to microbial infection such as papules, pustules and epidermal collarettes and on lesions associated with chronic inflammation and altered skin barrier, such as scales, seborrhoea or lichenification. The formulation as a spray permits to treat extensive area and is easy to use. The relatively low viscosity of the product makes it suitable for haired skin and its consistency permits great adherence to skin surface. These characteristics explain that close to $90 \%$ (89\%) of owners are very satisfied of the spray. Despite the good results obtained here, with a statistically significant improvement of both extent and severity of lesions after treatment, there are some limits in our study, notably its opened, uncontrolled character and the inclusion of dogs with various clinical presentations. Therefore, these very encouraging results need to be confirmed with further controlled studies in the field.

\section{Acknowledgements}

This study was carried out with the support of Laboratoire de Dermo-Cosmétique Animale (LDCA), Castres, France. The authors would like to thank Drs C. Anel, M. Bitsch, R. Butler, A. Cabrero, C. Chen, S. Chen, G. Duplessis, I. Esclapez Vegara, H.S. Han, K. Hofmann, I. Holz, YS. Hsiao, S. Jia, O. Koorts, J. Lee, M. Nagata, J. Neethling, M. Pacheco Duran, K. Paton, F. Schäfer, F. Shi, Z. Shi, D. Spith, S. White, B. Wicklein, I. Winkler, J. Xu, BJ. Yin, C. Zandri, P. Zeng, L. Zhang and D. Zhu for their cases included in the study.

\section{Conclusion}

These results confirm that the use of a topical treatment with a spray containing antimicrobial and antibiofilm agents may be of interest for the management of microbial infections in dogs on a clinical daily basis.

\section{References}

[1]. Hill P, Lo A, Can E, Smith DJ, Williams V, et al., (2006) Survey of the prevalence, diagnosis and treatment of dermatological conditions in small animal in general practice. Vet Record. 158(16): 533-539.

[2]. Beco L, Guaguère E, Lorente Mendez C, Noli C, Vroom M, et al., (2013) Suggested guidelines for using systemic antimicrobials in bacterial skin infections: part 2- antimicrobial choice, treatment regimens and compliance. Vet Record.172(6): 156-60.

[3]. Hillier A, Lloyd DH, Scott Weese J, Boothe D, Skyes JE, et al., (2014) Guidelines for the diagnosis and antimicrobial therapy in canine superficial bacterial folliculitis (antimicrobial guidelines working group of the International Society for Companion Animal Infectious Diseases). Vet Dermatol. 25(3): 163-175.

[4]. Mueller RS, Bergvall K, Bensignor E, Bond R (2003) A review of topical therapy for skin infections with bacteria and yeasts. Vet Dermatol. 23(4): 330-341.

[5]. Bensignor E, Fabries L, Bailleux L (2016) A split-body, randomized, blinded study to evaluate the efficacy of a topical spray composed of essential oils and essential fatty acids from plant extracts with antimicrobial properties. Vet Dermatol. 27(6): 464-e123.

[6]. Lloyd DH, Lamport AI (1999) Activity of chlorhexidine shampoos in vitro against Staphylococcus intermedius, Pseudomonas aeruginosa and Malassezia pachydermatis. Vet Record. 144: 536-537.

[7]. Murayama N, Nagata M, Terada Y, shibata S, Fukata T (2010) Efficacy of a surgical scrub including $2 \%$ chlorhexidine acetate for canine superficial pyoderma. Vet Dermatol. 21: 586-592.

[8]. Loeffler A, Cobb MA, Bond R (2011) Comparison of a chlorhexidine and a benzoyl peroxyde shampoo as sole treatment in canine superficial pyodermas. Vet Record. 169(10): 249-254.

[9]. Borio S, Colombo S, La Rosa G, De Lucia M, Damborq P, et al., (2015) Effectiveness of a combined ( $4 \%$ chlorhexidine digluconate shampoo and solution) protocol in MRS and non MRS canine superficial pyoderma: a randomized, blinded, antibiotic-controlled study. Vet Dermatol. 26(5): 339-344.

[10]. Bensignor E, Hann H, Guillot J (2012) Topical versus systemic treatment of Malassezia dermatitis in dogs: a comparative, randomized, blinded trial. Vet Dermatol. 23(1): 2-104.

[11]. Dinicola S, De Grazia S, Carlomagno G, Pintucci JP (2014) N-acetylcysteine as powerful molecule to destroy bacterial biofilms. A systematic review. Eur Rev Med Pharmacol Sci. 18(19): 2942-8.

[12]. May ER, Conklin KA, Bemis DA (2016) Antibacterial effect of N-acetylcysteine on common canine otits externa isolates. Vet Dermatol. 27(3): 19990.

[13]. Song CY, Nam E, Park SH, Hwang VY (2013) In vitro efficacy of the essential oil from Leptosperumscoparium (manuka) on antimicrobial susceptibility and biofilm formation in Staphylococcus pseudintermedius isolates form dogs. Vet Dermatol. 24(4): 404-e87.

[14]. Fratini F, Mancini S, Turchi B, Cerri D, Giusti G, et al., (2017) A novel interpretation of the Fractional Inhibitory Concentration Index: The case Origanum vulgare L. and Leptospermum scoparium J. R. et G. Forst essential oils against Staphylococcus aureus strains. Microbiol Res. 195: 11-17.

[15]. Thosar N, Basak S, Bahadure RN, Rajurkar M (2013) Antimicrobial efficacy of five essential oils against oral pathogens: An in vitro study. Eur J Dent. $7(1): 71-7$.

[16]. Chen CC, Yan SH, Yen MY, Wu PF, Wen ZH, et al., (2016) Investigations of kanuka and manuka essential oils for in vitro treatment of disease and cellular inflammation caused by infectious microorganisms. J Microbiol Immunol Infect. 49(1): 104-11.

[17]. Zuzarte M, Goncalves MJ, Cavaleiro C, Canhoto J, Silva LS, Silva MJ, et al., (2011) Chemical composition and antifungal activity of the essential oils of Lavandulaviridis. J Med Microbiol. 60(5): 612-18.

[18]. Oguz A, Uslukaya O, Alabalik U, Kapan M, Bozadaq Z, et al., (2015) Topical N-acetylcysteine improves wound healing comparable to dexpanthenol: an experimental study. Int Surg. 100(4): 656-61.

[19]. Nakai K, Yoneda K, Murakami Y, Koura A, Maeda R, et al., (2015) Effects of topical $\mathrm{N}$-acetylcysteine on skin hydration/transepidermal water loss in healthy volunteers and atopic dermatitis patients. Ann Dermatol. 27(4): 450-1.

[20]. Leizer C, Ribnicky D, Poulev A, Dushenkov S, Raskin I (2000) The Composition of Hemp Seed Oil and Its Potential as an Important Source of Nutrition. J Nutraceut Funct Med Food. 2(4): 35-53.

[21]. Olivry T, DeBoer DJ, Favrot C, Jackson HA, Nuttall T, et al., (2015) Treatment of canine atopic dermatitis: 2015 updated guidelines from the International Committee on Allergic Diseases of Animals (ICADA). Vet Res. 11 : 210-25. 